\section{Sugar-coated}

It's a tough truth to swallow, but studying what's inside a cell can be easier than characterizing its surface. However, Nagahori et al., writing in Biochemistry, have found a way to sweeten the deal for glycosphingolipidomics researchers. Their aim was to find an efficient method to probe the structure and function of cellsurface glycosphingolipids (GSLs), which play roles in cell adhesion and signaling. It's been described before that an aminooxy functional group can be linked to a GSL that has been treated with ozone to create a reaction-ready aldehyde group, and this "glycoblotting" process has been exploited for MS-based analysis.

Here, the authors adapt the technique to aminooxy-functionalized gold nanoparticles. With this method, it is possible to go from crude lipid fraction to MS-based structural profiling within a few hours. Excitingly, the GSL-studded nanoparticles may mimic the clusters of GSLs that are believed to play a role in interactions implicated in malignancy. For such studies, the GSL-conjugated nanoparticles were analyzed by surface plasmon resonance for binding with an immobi- lized GSL of interest. Although further data will be needed to validate the physiological relevance of nanoparticle-anchored "microdomains," this advance should open up cell surface molecules to studies as detailed as those performed on their intracellular cousins.

\section{Nagahori et al. Structural and func- tional glycosphingolipidomics by gly- coblotting with an aminooxy-function- alized gold nanoparticle. Biochemistry [Epub ahead of print, December 31, 2008, doi:10.1021/bi801640n].}

\section{Fresh Bait}

Many prey proteins have been identified in yeast two-hybrid assays, but the system falls short when it comes to higher-order complexes. One way to cast the net wider is to use yeast three-hybrid systems, in which a third "bridge" protein stabilizes the interaction between bait and prey. In such assays, an important test is to show that the reporter signal is dependent upon the expression of the third element, and for that reason, the bridge protein is expressed from an inducible promoter. Unfortunately, suboptimal control, such as promoter leakiness, can muddy the waters. Moreover, the inducer molecule may alter yeast responses, raising the specter of spurious findings. In a report in Nucleic Acids Research, Moriyoshi introduces a new system for assaying tripartite protein interactions in yeast, describing a battery of tests to determine a suitable regulatory system for tightly controlled induction.

The basic system uses cytomegalovirus (a weak promoter in yeast) to drive a Tet transactivator, and limits the promoter region for the bridge protein to a single Tet operator sequence. Although the addition of doxycycline is effective in turning off expression of this third protein, a low copy number version of the plasmid is also available for situations requiring a higher signal-to-noise ratio. These new yeast vectors should simplify capture of novel three-protein complexes, and may also be adaptable to assays aimed at identifying inhibitors of protein-protein interactions.

Moriyoshi. pBT, a novel vector for tetracycline-regulated yeast three-hybrid assay. Nucleic Acids Res. [Epub ahead of print, December 2, 2008, doi:10.1093/ nar/gkn969].

\title{
Forced Entry
}

From the point of view of a lentiviral vector, breaching a target cell under real-world conditions can be a formidable challenge. Hydrodynamic blood flow in vivo can whisk vector particles past would-be targets, and ex vivo transduction can require inhospitably low temperatures. Coupling magnetic nanoparticles to vector virions and applying a magnetic field has been shown to enhance adsorption and transduction. In an article appearing in $P N A S$, Hofmann et al. show that this approach can be extended to especially challenging transduction conditions such as targeting of the blood vessel lumen. In one test, mouse aortas being perfused ex vivo were exposed to lentiviral vector-magnetic nanoparticle (LV/MNP) conjugates, and a magnet was placed at one side of the vessel. In the area of strongest magnetic force, the LV/ MNPs overcame the hydrodynamic forces to deliver the vector into endothelial cells. Using the same system, the authors also demonstrated that LV/MNP-transduced human umbilical vein endothelial cells (HUVECs) could be directed to the endothelial layer. In vivo, a striking redistribution in relative transduction efficiency of liver versus lung was found in mice with a magnet immobilized at the right abdominal wall. Using the genetically modified HUVECs in vivo (this time in a carotid artery injury model) the authors also showed successful trans-

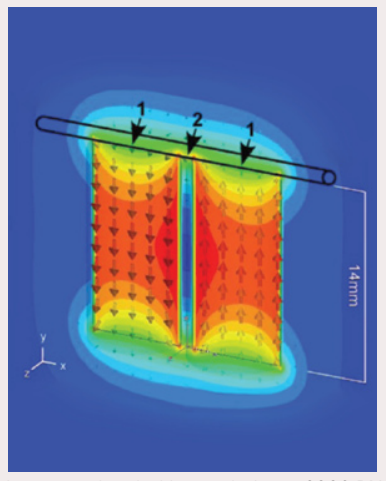

Image reprinted with permission (c) 2009 PNAS duction of the vessel in a magnet-dependent manner. As hoped, magnet-guided LV/MNPs can also overcome the inefficiencies of hypothermic conditions, a point driven home by successful transduction of aortic strips incubated at $4^{\circ} \mathrm{C}$. This work shows the increasing sophistication of lentiviral delivery systems, and suggests that a standard viral particle preparation could be targeted to different tissues or regions by simply altering the position of a guiding magnet.

Hofmann et al. 2009. Combined targeting of lentiviral vectors and positioning of transduced cells by magnetic nanoparticles. Proc Natl Acad Sci USA 106:44-49.

Selected and written by Nijsje Dorman, Ph.D. T[GA

BioTechniques 46:153 (March 2009) doi 10.2144/000113098 